\title{
A COMPARAÇÃO ENTRE MÉdIAS DE TRATAMENTOS NA ANÁLISE DA VARIÂNCIA
}

FREDERICO PIMENTEL GOMES

Escola Superior de Agricultura "Luiz de Queiroz", Universidade de São Paulo

\section{INDICE}

1 - 0 Problema a Resolver .. . . . . . . . . . . . . . . . . . . . 2

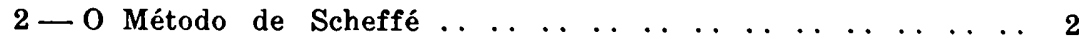

3- 0 Método de Tukey . . . . . . . . . . . . . . . . . . . . . . 6

4 - Propriedades dos Novos Testes $\ldots \begin{array}{llllllllll} & \ldots & \ldots & \ldots & \ldots & \ldots & \ldots & \ldots & \ldots & 7\end{array}$

5 - Agradecimento . . . . . . . . . . . . . . . . . . . . . . . . 11

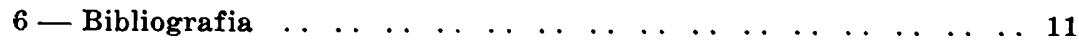


Anais da E. S. A. "Luiz de Queiroz"

\section{1 - O PROBLEMA A RESOLVER}

A comparação entre médias na análise da variância é um problema já bem velho, mas que até recentemente não tinha nenhuma solução satisfatória. Com efeito, o uso do teste $t$ não se justifica neste caso, a não ser para fazer algumas comparações escolhidas prèviamente, antes de examinar os dados do experimento, ou então quando êste encerra apenas dois tratamentos. FISHER (1, p. 58) propôs uma solução baseada no teste $t$, mas esta solução não é exata, pois se trata apenas de um método empírico bastante grosseiro. BRIEGER (9, pp. 490-491) introduziu também um método, igualmente baseado no teste $t$ e empírico, embora bastante razoável. DUNCAN (2 e 3) e TUKEY (4) estudaram posteriormente o assunto em trabalhos de grande interêsse. Mas foi só em 1952 que SCHEFFÉ (5) e TUKEY (6) lançaram as bases de dois métodos, ambos exatos, que serão de grande utilidade na análise estatística.

Neste artigo explicaremos como usar os novos processos de Scheffé e Tukey.

\section{2 - O METODO DE SCHEFFE}

Suponhamos um experimento com $k$ tratamentos e $r$ repetições, no qual obtivemos uma estimativa $s$ para o êrro residual com $n$ graus de liberdade. Então o intervalo de confiança ("confidence interval") para um contraste

$$
\mathrm{Y}=\mathrm{c} 1 \mathrm{y} 1+\mathrm{c} 2 \overline{\mathrm{y}} \mathbf{2}+\ldots+\mathrm{ck} \ddot{\mathrm{yk}}
$$

entre as médias de tratamentos, com probabilidade $\alpha$, é dado por

$(2,1) c 1 \bar{y} 1+c 2 \bar{y} 1+\ldots+c k y k+s l_{r}^{\prime(k-1) \bar{F}}\left(c_{1}{ }^{2}+c_{2}{ }^{2}+\ldots+c k^{2}\right)$

onde $\mathrm{F}$ é o valor de $\mathrm{F}$ da tábua, para a probabilidade " (geralmente $5 \%$ ou $1 \%$ ) com graus de liberdade $k-1$ e $n$, e onde se exige 


$$
\mathrm{c1}+\mathrm{c2} \ldots+\mathrm{ck}=0 .
$$

Se se preferir usar $\vartheta=V$ Fem lugar de F, a fórmula $(2,1)$ se transformará em

$(2,2) \mathrm{c} 1 \overline{\mathrm{y}} 1+\mathrm{c} 2 \overline{\mathrm{y}} 2+\ldots+\mathrm{ck} \overline{\mathrm{y}} \mathrm{k} \pm \mathrm{s}, \sqrt{\frac{\mathrm{k}-1}{\mathrm{r}}\left(\mathrm{c}_{1}{ }^{2}+\mathrm{c}_{2}{ }^{2}+\ldots+\mathrm{ck}^{2}\right)}$

No caso da comparação de duas médias y y e ȳj temos

$$
(2,3) \quad \bar{y} i-\overline{y j} \pm s \quad \frac{2(k-1) F}{r}
$$

ou

$$
(2,4) \quad \overline{y i}-\overline{y j} \pm s \text { i) } \frac{}{\frac{2(k-1)}{r}}
$$

pois então

$$
\mathrm{c}_{1}^{2}+\mathrm{c}_{2}^{2}+\ldots+\mathrm{ck}^{2}=2 .
$$

Por exemplo, para $\mathrm{k}=8$ tratamentos, $\mathrm{r}=6$ repetições, $u=5 \%, \mathrm{~s}=7,2, \mathrm{com} 35$ graus de liberdade, achamos $\vartheta=1,51$, logo

$$
\overline{y i}-\overline{y j} \pm 7,2 \times 1,51 \quad l^{\prime} \frac{\overline{2} \div 7}{6}
$$

isto é,

$$
\bar{y} \mathbf{i}-\bar{y} j \pm 16,6
$$

Suponhamos que yi $=184,5$ e $\ddot{y} j=161,3$ são duas médias quaisquer obtidas (podem ser a maior e a menor, por exemplo). Temos $184,5-161,3=23,2$ e o intervalo de confiança será dado por $23,2 \pm 16,6$, isto é, terá extremos 6,6 e 39,8 , logo temos 
95\% de probabilidade de que a verdadeira diferença entre essas médias esteja entre 6,6 e 39,8 .

Tôda diferença entre duas médias maior que 16,6 será significativa ao nível de $5 \%$.

No experimento que estamos discutindo as médias foram, em ordem decrescente, as seguintes:

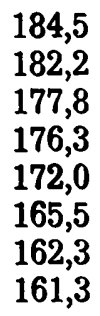

Vemos, pois, que a maior média $(184,5)$ difere significativamente das três menores $(165,5 ; 162,3$ e 161,3$)$. O mesmo acontece com a segunda média da tabela acima $(182,2)$. Já a terceira $(177,8)$ difere significativamente apenas das duas menores $(162,3$ e 161,3$)$.

Suponhamos, agora, que queremos comparar a maior média com a média de todos os outros tratamentos. Consideramos a função linear

$$
\begin{aligned}
\mathrm{Y} & =\overline{\mathrm{y}} 1-\frac{1}{7} \overline{\mathrm{y}} 2-\frac{1}{7} \overline{\mathrm{y}} 3 \ldots-\frac{1}{7}-\frac{\mathrm{y} 8}{7}(\mathrm{y} 2+\overline{\mathrm{y}} 3 \ldots+\overline{\mathrm{y}} 8) \\
& =\overline{\mathrm{y}} 1-\frac{1}{7}
\end{aligned}
$$

A estimativa do contraste $Y$ é, no caso em questão,

$$
\begin{aligned}
Y & =184,5-\frac{1}{7}(182,2+177,8+\ldots+161,3) \\
& =13,4 .
\end{aligned}
$$



por

E, de acôrdo com $(2,2)$, o intervalo de confiança será dado

$$
13,4 \pm 7,2 \times 1,51 \sqrt{\frac{7}{6}\left(1+7 \times \frac{1}{49}\right)}
$$

ou

$$
13,4 \pm 12,5
$$

Temos, pois, uma probabilidade de $95 \%$ de que o verdadeiro valor da diferença esteja entre $13,4-12,5=0,9$ e $13,4+$ $+12,5=25,9$. Como a diferença obtida $(13,4)$ é maior do que 12,5 , ela é significativa ao nível de $5 \%$ de probabilidade.

Qualquer outra comparação que interesse poderá ser estudada do mesmo modo. Por exemplo, poderemos comparar os quatro tratamentos de maior média com os outros quatro. Temos

$Y=\frac{1}{4}\left(\overline{y_{1}}+\overline{y_{2}}+\overline{y_{3}}+\overline{y_{4}}\right)-\frac{1}{4}\left(\bar{y}_{5}+\bar{y}_{6 i}+\overline{y_{7}}+\overline{y_{8}}\right)=14.9$.

O intervalo de confiança é dado por

$$
14,9 \pm 7,2 \times 1,51 \sqrt{\frac{7}{6}\left(4 \div \frac{1}{16}+4 \div \frac{1}{16}\right)}
$$

ou

$$
14,9 \pm 8,3
$$

A verdadeira diferença estará, pois, entre $14,9-8,3==6,6$ e $14,9+8,3=23,2$ com $95 \%$ de probabilidade. Qualquer diferença semelhante calculada (diferença entre as médias de quatro dos tratamentos e a média dos quatro restantes) ć significativa ao nivel de $5 \%$ se exceder 8,3 . No caso vertente a diferença foi $14,9>8,3$ e é, pois, significativa. 


\section{3 - O MÉTODO DE TUKEY}

Este método se baseia na amplitude total "estudentizada" ("studentized range") e se aplica de preferència ao caso da comparação de duas médias, escolhidas entre as $k$ médias de tratamentos, todos com o mesmo número de repetições. Suponhamos, pois, que temos ainda $k$ tratamentos e $r$ repetições, e uma estimativa $s$ para o êrro residual, com $n$ graus de liberdade. Então o intervalo de confiança para uma diferença yi - yj entre duas médias de tratamentos é dada por

$$
\bar{y} i-\bar{y} j \pm \frac{s}{V^{---r}} q
$$

onde $q$ é valor da amplitude total "estudentizada" para o nível $u$ de probabilidade (geralmente $5 \%$ ou $1 \%$ ), para o caso de $k$ tratamentos e $n$ graus de liberdade para a estimativa do êrro. Tábuas de $q$ podem ser encontradas em DIXON e MASSEY (7). Aí, porém, aparecem incorreções, que foram corrigidas por MAY (8). As incorreções são de pouca monta na tábua referente a $a=5 \%$, mas são bem ponderáveis no caso de $a=1 \%$.

Suponhamos $s=7,2, k=8$ tratamentos, $r=6$ repetições, ll $=5 \%$, como anteriormente, e teremos:

$$
\overline{y i}-\overline{y j} \pm \frac{7,2}{\sqrt{\overline{6}}} \quad 4,56=\bar{y} i-\bar{y} j \pm 13,4
$$

Para $\vec{y} 1=184,5$, yj $=161,3$, temos $184,5-161,3=23,2$ e o intervalo de confiança terá os extremos $23,2-13,4=9,8$ e $23,2+13,4=36,6$. Note-se que êste intervalo é menor do que obtido pelo método de Scheffé. Isto se deve a têrmos, no caso do método de Tukey, uma hipótese menos geral. Para a comparação de médias duas a duas o processo de Tukey, baseado na amplitude total "estudentizada", é, pois, preferível. Mas o método de Scheffé, aplicável sempre que caiba o teste F, é de uso muito mais generalizado. 
Se as médias são as mesmas do capítulo anterior, vemos pelo método de Tukey que a mais elevada $(184,5)$ diferirá significativamente, ao nível de $5 \%$, de tôdas as médias que forem inferiores a $184,5-13,4=171,1$. Difere significativamente, pois, como antes, de 165,$5 ; 162,3$ e 161,3 . O verdadeiro valor da diferença $184,5-165,5=19,0$ estará, portanto, com $95 \%$ de probabilidade, entre $19,0-13,4=5,6$ e $19,0+13,4=32,4$.

\section{4 - PROPRIEDADES DOS NOVOS TESTES}

Tanto o teste de Tukey como o de Scheffé só devem ser postos em prática quando o valor de $F$ (ou de " i) obtido na análise da variância fôr significativo, pois, se isto não se der, nenhuma das diferenças entre médias poderá ser significativa. A recíproca, porem, não é verdadeira, isto é, o fato de o $F$ (ou v ) da análise da variância ser significativo não exige que haja duas médias diferindo significativamente, mas sim que haja um contraste entre as médias de tratamentos que seja significativo. Este contraste poderá ser de natureza bastante complexa e talvez sem nenhum interêsse prático.

Suponhamos, por exemplo, um experimento com $\mathrm{k}=3$ tratamentos, $r=5$ repetiçōes, $\mathrm{s}^{2}=1,70$, com 12 graus de liberdade. Sejam 10, 8 e 8 as médias dos três tratamentos. A análise da variância é, então, a que damos abaixo

\begin{tabular}{l|c|c|c|c}
\hline Causa de variação & G. L. & $\begin{array}{c}\text { Soma dess } \\
\text { Quadradss }\end{array}$ & $\begin{array}{c}\text { Quadrado } \\
\text { médio }\end{array}$ & $n$ \\
\hline Tratamentos & 2 & 13,3 & 6,65 & $1,98 *$ \\
Residuo & 12 & 20,4 & 1,70 & - \\
Total & 14 & 33,7 & - & - \\
\hline
\end{tabular}

Para julgar a diferença entre duas médias o método de Scheffé nos dá então o valor

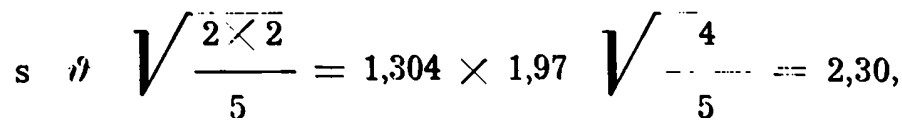


e, no caso do método de Tukey,

$$
\frac{1,304}{\sqrt{5}} 3,77-2,20,
$$

ambas ao nivel de $5 \%$ de probabilidade.

Como a maior diferença entre as médias é igual a 2, não pode ser significativa. No entanto a função linear.

$$
\mathrm{Y}=\frac{2}{\sqrt{3}} \overline{\mathrm{y}} 1-\frac{1}{\sqrt{3}} \overline{\mathrm{y}} 2-\frac{1}{\sqrt{3}} \overline{\mathrm{y}} 3=-\frac{2 \overline{\mathrm{y}} 1-\overline{\mathrm{y}} 2-\overline{\mathrm{y}} 3}{\sqrt{3}}
$$

que é um contraste, pois a soma dos coeficientes é nula, nos dá

$$
c_{1}^{2}+c_{2}^{2}+c_{3}^{2}=-\frac{4}{3}+\frac{1}{3}+\frac{1}{3}=2,
$$

e, pois, o intervalo de confiança pelo método de Scheffé será

$$
\mathrm{Y}=2,30 \text {. }
$$

E como os valores observados nus dão

$$
\mathrm{Y}=\frac{2 \backslash 10-8-8}{V^{-}-8}=2,31,
$$

vemos que a diferença entre a maior média e as outras duas é significativa ao nível de $5 \%$, o que justifica o resultado obtido na análise da variância.

Um caso semelhante a êste ocorreu com um experimento de adubação de cana com adubos verdes realizado pela Usina Monte Alegre, em Piracicaba. O experimento, em quadrado latino de $6 \times 6$, teve a seguinte análise da variância. 


\begin{tabular}{|c|c|c|c|c|}
\hline Causa de variação & G. L. & $\begin{array}{l}\text { Soma dos } \\
\text { quadrados }\end{array}$ & $\begin{array}{l}\text { Quadrado } \\
\text { médio }\end{array}$ & i) \\
\hline $\begin{array}{l}\text { Tratamentos } \\
\text { Linhas } \\
\text { Colunas } \\
\text { Resíduo }\end{array}$ & $\begin{array}{r}5 \\
5 \\
5 \\
20\end{array}$ & $\begin{array}{l}37.969 \\
52.972 \\
41.183 \\
50.976\end{array}$ & $\begin{array}{r}7.594 \\
10.594 \\
8.237 \\
2.549\end{array}$ & $\begin{array}{l}1,72 * \\
2,04 * * \\
1,80 * \\
-\end{array}$ \\
\hline Total & 35 & 183.100 & - & 一 \\
\hline
\end{tabular}

As médias dos tratamentos foram:

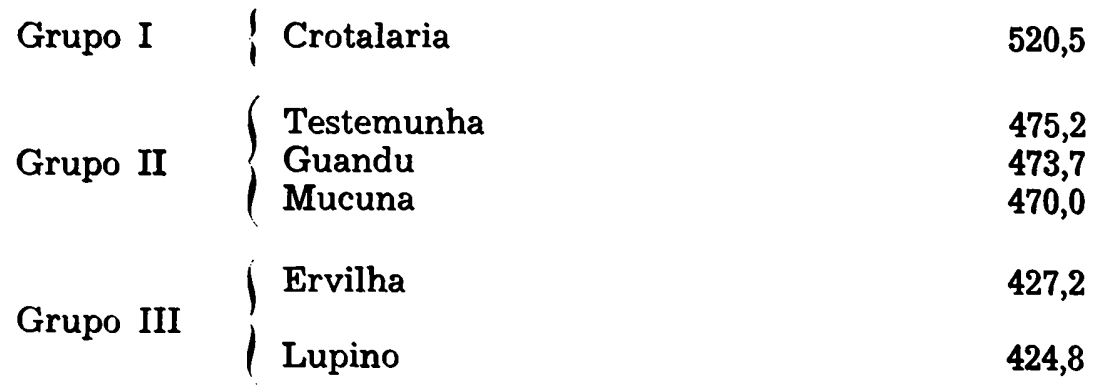

Para julgar a diferença entre duas dessas médias o método de Tukey nos dá, ao nível de $5 \%$, o valor

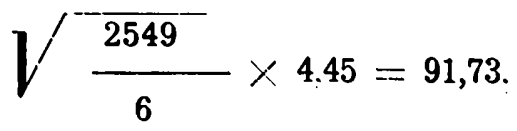

Como a diferença entre a maior média e as duas menores excede êste número, conclui-se que a crotalária é significativamente superior à ervilha e ao lupino, ao nivel de $5 \%$.

Se usarmos, porém, o método de Scheffé, teremos

$$
V \overline{2549 \times 1,64} V \frac{\overline{5} \times 2}{6}=106,90
$$


Logo, por êste critério, nenhuma das diferenças entre médias seria significativa. Podemos, porém, grupar os tratamentos em três grupos, I, II e III, conforme indicado acima. Então a comparação entre os grupos I e III seria dada pela função

$$
\left.\bar{y} 1-\frac{1}{2} \overline{(\bar{y} 5}+\bar{y} 6\right)=520,5-\frac{427,2+424,8}{2}=94,5,
$$

para a qual o método de Scheffé nos dá

$$
\sqrt{2549} \quad 1,64 V^{\prime} \frac{5}{6}\left(1+\frac{1}{4}+\frac{1}{4}\right)=87,28 .
$$

O grupo I é, pois, significativamente superior ao grupo III, ao nível de $5 \%$. Já os grupos I e II não diferem significativamente entre si, como o leitor poderá verificar.

E' interessante salientar que os grupos II e III diferem significativamente, ao nível de $5 \%$ de probabilidade, se utilizarmos o teste $t$. Mas êste teste não é válido numa comparação como esta, e o teste exato de Scheffé mostra que a diferença entre as médias dêsses dois grupos, igual a 47,0 e correspondente ao contraste

$$
\frac{1}{3}(\overline{\mathrm{y}} 2+\overline{\mathrm{y}} 3+\overline{\mathrm{y}} 4)-\frac{1}{2}(\overline{\mathrm{y}} 5+\mathrm{y} \overline{6}) .
$$

só seria significativa ao nível de $5 \%$ se excedesse o valor

$$
\sqrt{2549} \quad 1,64 \sqrt{\frac{5}{6}\left(3 ; \frac{1}{9}+2 \times \frac{1}{4}\right)}=69,0 .
$$

No caso de não ser o mesmo o número de repetições para todos os tratamentos, as fórmulas $(2,1)$ e $(2,2)$ devem ser modificadas. Com efeito, se o primeiro tratamento tem $r 1$ repetições, 
o segundo, r2 repetições, e assim por diante, as novas fórmulas serão :

$(4,1) \mathrm{cl} \overline{\mathrm{y}} 1+\mathrm{c} 2 \overline{\mathrm{y}} 2+\ldots+\mathrm{ck} \overline{\mathrm{y}} \mathrm{k} \pm \mathrm{s} V(\mathrm{k}-1) \mathrm{F}\left(\frac{\mathrm{c}_{1}{ }^{2}}{\mathrm{rl}}+\frac{\mathrm{c}_{2}{ }^{2}}{\mathrm{r}^{2}}+\ldots+\frac{\mathrm{ck}^{2}}{\mathrm{rk}}\right)$ ou

$(4,2) c 1 \bar{y} 1+c 2 \bar{y} 2+\ldots+c k \bar{y} k \pm s i / \sqrt{(k-1)\left(\frac{c_{1}{ }^{2}}{r l}+\frac{c_{2}{ }^{2}}{r 2} \mid \ldots+\frac{c k^{2}}{r k}\right)}$

Convém assinalar também que no caso de as médias de tratamentos serem correlacionadas, o método de Tukey conduz a uma fórmula um pouco diferente da que vimos acima. Com efeito, sendo $\varrho$ o coeficiente de correlação entre dois tratamentos quaisquer, sempre o mesmo para qualquer par considerado, então o intervalo de confiança é dado por

$$
\bar{y} i-\bar{y} j \pm \frac{s q}{\sqrt{r}} \quad V \overline{1-0} .
$$

Tal é o caso, por exemplo, quando se tem um experimento em blocos incompletos equilibrados.

\section{5 - AGRADECIMENTO}

Agradecemos cordialmente aos nossos colegas Drs. Eno Miranda Cardoso e Nelson Camolese a autorização dada para utilizar neste artigo dados de um dos experimentos realizados por êles na Usina Monte Alegre.

\section{6 - BIBLIOGRAFIA}

1-FISHER, R. A. - "The Design of Experiments", sexta edição. Hafner Publishing Company, Nova York, 1951.

2-DUNCAN, D. B. - "A Significance Test for Differences Between Ranked Treatments in an Analysis of Variance". The Virginia Journal of Science 2: 171-189, 1951. 
3- - "On the Properties of the Multiple Comparisons Test". The Virginia Journal of Science $3: 49-67,1952$.

4-TUKEY, John W. - "Comparing Individual Means in the Analysis of Variance". Biometrics 5 : 99-114, 1949.

5-SCHEFFE, Henry - "A Method for Judging all Contrasts in the Analysis of Variance" (mimeografado). Columbia University, Nova York, 1952.

6- TUKEY, John W. - "Reminder Sheets for Discussion of Paper on Multiple Comparisons" (mimeografado).

7 - DIXON, Wilfrid J. e Frank J. Massey Jr. - "Introduction to Statistical Analysis". McGraw - Hill Book Company, Nova York, 1951.

8-MAY, Joyce M. - "Extended and corrected tables of the upper percentage points of the "Studentized" range". Biometrika 39 : 192-193, 1952.

9-BRIEGER, F. G. - "Limites unilaterais e bilaterais na análise estatística". Bragantia 6: 479-545, 1946. 
Tábua dos Valores da Amplitude Total Estudentizada, ao Nível de $5 \%$ de Probabilidade, computada par
$\left(\mathrm{k}=\mathrm{n}^{\circ}\right.$. de tratamentos; $\mathrm{n}=\mathrm{n}^{\circ}$. de graus de liberdade do êrro residual)

\begin{tabular}{|c|c|c|c|c|c|c|c|c|c|c|c|c|c|c|c|c|c|c|c|}
\hline $\mathbf{k}_{\mathrm{i}}$ & 2 & 3 & 4 & 5 & 6 & 7 & 8 & 9 & 10 & 11 & 12 & 13 & 14 & 15 & 16 & 17 & 18 & 19 & 20 \\
\hline $\begin{array}{l}1 \\
2 \\
3 \\
1\end{array}$ & $\begin{array}{r}18,0 \\
6,09 \\
4,50 \\
3,93\end{array}$ & $\begin{array}{c}26,7 \\
8,28 \\
5,88 \\
5,00\end{array}$ & $\begin{array}{c}32,8 \\
9,80 \\
6,83 \\
5,76\end{array}$ & $\begin{array}{r}37,2 \\
10,89 \\
7,51 \\
6,31\end{array}$ & $\begin{array}{r}40,5 \\
11,73 \\
8,04 \\
6,73\end{array}$ & $\begin{array}{r}43,1 \\
12,43 \\
8,47 \\
7,06\end{array}$ & $\begin{array}{r}45,4 \\
13,03 \\
8,85 \\
7,35\end{array}$ & $\begin{array}{c}47,3 \\
13,54 \\
9,18 \\
7,60\end{array}$ & $\begin{array}{r}49,1 \\
13,99 \\
9,46 \\
7,83\end{array}$ & $\begin{array}{c}50,6 \\
14,39 \\
9,72 \\
8,03\end{array}$ & $\begin{array}{r}51,9 \\
14,75 \\
9,95 \\
8,21\end{array}$ & $\begin{array}{r}53,2 \\
15,08 \\
10,16 \\
8,37\end{array}$ & $\begin{array}{l}54,3 \\
15,38 \\
10,35 \\
8,52\end{array}$ & $\begin{array}{l}55,4 \\
15,65 \\
10,52 \\
8,67\end{array}$ & $\begin{array}{l}56,3 \\
15,91 \\
10,69 \\
8,80\end{array}$ & $\begin{array}{l}57,2 \\
16,14 \\
10,84 \\
8,92\end{array}$ & $\begin{array}{r}58,0 \\
16,36 \\
10,98 \\
9,03\end{array}$ & $\begin{array}{c}58,8 \\
16,57 \\
11,12 \\
9,14\end{array}$ & $\begin{array}{r}59,6 \\
16,77 \\
11,24 \\
9,24\end{array}$ \\
\hline $\begin{array}{l}5 \\
6 \\
7 \\
8 \\
3\end{array}$ & $\begin{array}{l}3,61 \\
3,46 \\
3,34 \\
3,26 \\
3,20\end{array}$ & $\begin{array}{l}4,54 \\
4,34 \\
4,16 \\
4,04 \\
3,95\end{array}$ & $\begin{array}{l}5,18 \\
4,90 \\
4,68 \\
4,53 \\
4,42\end{array}$ & $\begin{array}{l}5,64 \\
5,31 \\
5,06 \\
4,89 \\
4,76\end{array}$ & $\begin{array}{l}5,99 \\
5,63 \\
5,35 \\
5,17 \\
5,02\end{array}$ & $\begin{array}{l}6,28 \\
5,89 \\
5,59 \\
5,40 \\
5,24\end{array}$ & $\begin{array}{l}6,52 \\
6,12 \\
5,80 \\
5,60 \\
5,43\end{array}$ & $\begin{array}{l}6,74 \\
6,32 \\
5,99 \\
5,77 \\
5,60\end{array}$ & $\begin{array}{l}6,93 \\
6,49 \\
6,15 \\
5,92 \\
5,74\end{array}$ & $\begin{array}{l}7,10 \\
6,65 \\
6,29 \\
6,05 \\
5,87\end{array}$ & $\begin{array}{l}7,25 \\
6,79 \\
6,42 \\
6,18 \\
5,98\end{array}$ & $\begin{array}{l}7,39 \\
6,92 \\
6,54 \\
6,29 \\
6,09\end{array}$ & $\begin{array}{r}7,52 \\
7,04 \\
6,65 \\
6,39 \\
6,19\end{array}$ & $\begin{array}{l}7,64 \\
7,14 \\
6,75 \\
6,48 \\
6,28\end{array}$ & $\begin{array}{l}7,75 \\
7,24 \\
6,84 \\
6,57 \\
6,36\end{array}$ & $\begin{array}{l}7,86 \\
7,34 \\
6,93 \\
6,65 \\
6,44\end{array}$ & $\begin{array}{l}7,95 \\
7,43 \\
7,01 \\
6,73 \\
:, 51\end{array}$ & $\begin{array}{l}8,04 \\
7,51 \\
7,08 \\
6,80 \\
6,58\end{array}$ & $\begin{array}{l}8,13 \\
7,59 \\
7,16 \\
6,87 \\
6,65\end{array}$ \\
\hline $\begin{array}{l}10 \\
11 \\
12 \\
13 \\
14\end{array}$ & $\begin{array}{l}3,15 \\
3,11 \\
3,08 \\
3,06 \\
3,03\end{array}$ & $\begin{array}{l}3,88 \\
3,82 \\
3,77 \\
3,73 \\
3,70\end{array}$ & $\begin{array}{l}4,33 \\
4,26 \\
4,20 \\
4,15 \\
4,11\end{array}$ & $\begin{array}{l}4,66 \\
4,58 \\
4,51 \\
4,46 \\
4,41\end{array}$ & $\begin{array}{l}4,91 \\
4,82 \\
4,75 \\
4,69 \\
4,64\end{array}$ & $\begin{array}{l}5,12 \\
5,03 \\
4,95 \\
4,88 \\
4,83\end{array}$ & $\begin{array}{l}5,30 \\
5,20 \\
5,12 \\
5,05 \\
4,99\end{array}$ & $\begin{array}{l}5,46 \\
5,35 \\
5,27 \\
5,19 \\
5,13\end{array}$ & $\begin{array}{l}5,60 \\
5,49 \\
5,40 \\
5,32 \\
5,25\end{array}$ & $\begin{array}{l}5,72 \\
5,61 \\
5,51 \\
5,43 \\
5,36\end{array}$ & $\begin{array}{l}\mathbf{5 , 8 3} \\
5,71 \\
5,61 \\
5,53 \\
\mathbf{5 , 4 6}\end{array}$ & $\begin{array}{l}5,93 \\
5,81 \\
5,71 \\
5,63 \\
5,56\end{array}$ & $\begin{array}{l}6,03 \\
5,90 \\
5,80 \\
5,71 \\
5,64\end{array}$ & $\begin{array}{l}6,12 \\
5,98 \\
5,88 \\
5,79 \\
5,72\end{array}$ & $\begin{array}{l}6,20 \\
6,06 \\
5,95 \\
5,86 \\
5,79\end{array}$ & $\begin{array}{l}6,27 \\
6,14 \\
6,02 \\
5,93 \\
5,86\end{array}$ & $\begin{array}{l}6,34 \\
6,20 \\
6,09 \\
6,00 \\
5,92\end{array}$ & $\begin{array}{l}6,41 \\
6,27 \\
6,15 \\
6,06 \\
5,98\end{array}$ & $\begin{array}{l}6,47 \\
6,33 \\
6,21 \\
6,11 \\
6,03\end{array}$ \\
\hline $\begin{array}{l}15 \\
16 \\
17 \\
18 \\
19\end{array}$ & $\begin{array}{l}3,01 \\
3,00 \\
2,98 \\
2,97 \\
2,96\end{array}$ & $\begin{array}{l}3,67 \\
3,65 \\
3,62 \\
3,61 \\
3,59\end{array}$ & $\begin{array}{l}4,08 \\
4,05 \\
4,02 \\
4,00 \\
3,98\end{array}$ & $\begin{array}{l}4,37 \\
4,34 \\
4,31 \\
4,28 \\
4,26\end{array}$ & $\begin{array}{l}4,59 \\
4,56 \\
4,52 \\
4,49 \\
4,47\end{array}$ & $\begin{array}{l}4,78 \\
4,74 \\
4,70 \\
4,67 \\
4,64\end{array}$ & $\begin{array}{l}4,94 \\
4,90 \\
4,86 \\
4,83 \\
4,79\end{array}$ & $\begin{array}{l}5,08 \\
5,03 \\
4,99 \\
4,96 \\
4,92\end{array}$ & $\begin{array}{l}5,20 \\
5,15 \\
5,11 \\
5,07 \\
5,04\end{array}$ & $\begin{array}{l}5,31 \\
5,26 \\
5,21 \\
5,17 \\
5,14\end{array}$ & $\begin{array}{l}5,40 \\
5,35 \\
5,31 \\
5,27 \\
5,23\end{array}$ & $\begin{array}{l}5,49 \\
5,44 \\
5,39 \\
5,35 \\
5,32\end{array}$ & $\begin{array}{l}\mathbf{5 , 5 7} \\
5,52 \\
5,47 \\
5,43 \\
5,39\end{array}$ & $\begin{array}{l}5,65 \\
5,59 \\
5,55 \\
5,50 \\
5,46\end{array}$ & $\begin{array}{l}5,72 \\
5,66 \\
5,61 \\
5,57 \\
5,53\end{array}$ & $\begin{array}{l}5.79 \\
5,73 \\
5,68 \\
5.63 \\
5,59\end{array}$ & $\begin{array}{l}5,85 \\
5,79 \\
5,74 \\
5,69 \\
5,65\end{array}$ & $\begin{array}{l}5,91 \\
5,84 \\
5,79 \\
5,74 \\
5,70\end{array}$ & $\begin{array}{l}\mathbf{5 , 9 6} \\
\mathbf{5 , 9 0} \\
\mathbf{5 , 9 4} \\
\mathbf{5 , 7 . 9} \\
\mathbf{5 , 7 5}\end{array}$ \\
\hline $\begin{array}{l}20 \\
24 \\
30 \\
40\end{array}$ & $\begin{array}{l}2,95 \\
2,92 \\
2,89 \\
2,86\end{array}$ & $\begin{array}{l}3,58 \\
3,53 \\
3,48 \\
3,44\end{array}$ & $\begin{array}{l}3,96 \\
3,90 \\
3,94 \\
3,79\end{array}$ & $\begin{array}{l}4,24 \\
4,17 \\
4,11 \\
4,04\end{array}$ & $\begin{array}{l}4,45 \\
4,37 \\
4,30 \\
4,23\end{array}$ & $\begin{array}{l}4,62 \\
4,54 \\
4,46 \\
4,39\end{array}$ & $\begin{array}{l}4,77 \\
4,68 \\
4,60 \\
4,52\end{array}$ & $\begin{array}{l}4,90 \\
4,81 \\
4,72 \\
4,63\end{array}$ & $\begin{array}{l}5,01 \\
4,92 \\
4,83 \\
4,74\end{array}$ & $\begin{array}{l}5,11 \\
5,01 \\
4,92 \\
4,82\end{array}$ & $\begin{array}{l}5,20 \\
5,10 \\
5,00 \\
4,90\end{array}$ & $\begin{array}{l}5,28 \\
5,18 \\
5,08 \\
4,98\end{array}$ & $\begin{array}{l}5,36 \\
5,25 \\
5,15 \\
5,05\end{array}$ & $\begin{array}{l}5,43 \\
5,32 \\
5,21 \\
5,11\end{array}$ & $\begin{array}{l}5,50 \\
5,38 \\
5,27 \\
5,17\end{array}$ & $\begin{array}{l}5,56 \\
5.44 \\
5,33 \\
5,22\end{array}$ & $\begin{array}{l}\mathbf{5 , 6 1} \\
5,50 \\
5,38 \\
5,27\end{array}$ & $\begin{array}{l}\mathbf{5 , 6 6} \\
5,55 \\
5,43 \\
5,32\end{array}$ & $\begin{array}{l}5,71 \\
5,59 \\
5,48 \\
5,36\end{array}$ \\
\hline $\begin{array}{r}60 \\
120 \\
\infty\end{array}$ & $\begin{array}{l}2,83 \\
2,80 \\
2,77\end{array}$ & $\begin{array}{l}3,40 \\
3,36 \\
3,32\end{array}$ & $\begin{array}{l}3,74 \\
3,69 \\
3,63\end{array}$ & $\begin{array}{l}3,98 \\
3,92 \\
3,86\end{array}$ & $\begin{array}{l}4,16 \\
4,10 \\
4,03\end{array}$ & $\begin{array}{l}4,31 \\
4,24 \\
4,17\end{array}$ & $\begin{array}{l}4,44 \\
4,36 \\
4,29\end{array}$ & $\begin{array}{l}4,55 \\
4,47 \\
4,39\end{array}$ & $\begin{array}{l}4,65 \\
4,56 \\
4,47\end{array}$ & $\begin{array}{l}4,73 \\
4,64 \\
4,55\end{array}$ & $\begin{array}{l}4,81 \\
4,71 \\
4,62\end{array}$ & $\begin{array}{l}4,88 \\
4,78 \\
4,68\end{array}$ & $\begin{array}{l}4,94 \\
4,84 \\
4,74\end{array}$ & $\begin{array}{l}5,00 \\
4,90 \\
4,80\end{array}$ & $\begin{array}{l}5.06 \\
4.95 \\
4.84\end{array}$ & $\begin{array}{l}5,11 \\
5,00 \\
4,89\end{array}$ & $\begin{array}{l}5,15 \\
5,04 \\
4,93\end{array}$ & $\begin{array}{l}5,20 \\
5,09 \\
4,97\end{array}$ & $\begin{array}{l}5,24 \\
5,13 \\
5,01\end{array}$ \\
\hline
\end{tabular}


Tábua dos Valores da Amplitude Total Estudentizada, ao Nivel de $1 \%$ í de Probabilidade, computada por Joyce M. MAY (8)

\begin{tabular}{|c|c|c|c|c|c|c|c|c|c|c|c|c|c|c|c|c|c|c|c|}
\hline$k$ & 2 & 3 & 4 & 5 & 6 & 7 & 8 & 9 & 10 & 11 & 12 & 13 & 14 & 15 & 16 & 17 & 18 & 19 & 20 \\
\hline $\begin{array}{l}1 \\
2 \\
3 \\
4\end{array}$ & $\begin{array}{c}90,0 \\
14,0 \\
8,26 \\
6,51\end{array}$ & $\begin{array}{c}134 \\
18,9 \\
10,56 \\
8,08\end{array}$ & $\begin{array}{c}164 \\
22,3 \\
12,17 \\
9,17\end{array}$ & $\begin{array}{r}186 \\
24,7 \\
13,34 \\
9,97\end{array}$ & $\begin{array}{c}202 \\
26,6 \\
14,25 \\
10,58\end{array}$ & $\begin{array}{c}216 \\
28,2 \\
15,00 \\
11,10\end{array}$ & $\begin{array}{c}227 \\
29,5 \\
15,65 \\
11,55\end{array}$ & $\begin{array}{c}237 \\
30,7 \\
16,20 \\
11,93\end{array}$ & $\begin{array}{c}246 \\
31,7 \\
16,69 \\
12,26\end{array}$ & $\begin{array}{c}253 \\
32,6 \\
17,13 \\
12,56\end{array}$ & $\begin{array}{l}260 \\
33,4 \\
17.53 \\
\mathrm{i} 2,84\end{array}$ & $\begin{array}{c}266 \\
34,2 \\
17,89 \\
13,09\end{array}$ & $\begin{array}{c}272 \\
34,8 \\
18,23 \\
13,32\end{array}$ & $\begin{array}{c}277 \\
35,5 \\
18,54 \\
13,53\end{array}$ & $\begin{array}{c}282 \\
36,0 \\
18,83 \\
13,73\end{array}$ & $\begin{array}{l}286 \\
36,5 \\
19,09 \\
13,92\end{array}$ & $\begin{array}{c}291 \\
37,0 \\
19.33 \\
14,09\end{array}$ & $\begin{array}{c}295 \\
37,5 \\
19,56 \\
14,25\end{array}$ & $\begin{array}{c}298 \\
38,0 \\
19,79 \\
14,40\end{array}$ \\
\hline $\begin{array}{l}5 \\
6 \\
7 \\
8 \\
9\end{array}$ & $\begin{array}{l}5,62 \\
5,24 \\
4,94 \\
4,74 \\
4,60\end{array}$ & $\begin{array}{l}6,83 \\
6,32 \\
5,89 \\
5,63 \\
5,42\end{array}$ & $\begin{array}{l}7,65 \\
7,03 \\
6,52 \\
6,20 \\
5,96\end{array}$ & $\begin{array}{l}8,26 \\
7,56 \\
6,98 \\
6,63 \\
6,35\end{array}$ & $\begin{array}{l}8,73 \\
7,97 \\
7,35 \\
6,96 \\
6,66\end{array}$ & $\begin{array}{l}9,12 \\
8,31 \\
7,65 \\
7,24 \\
6,91\end{array}$ & $\begin{array}{l}9,46 \\
8,61 \\
7,91 \\
7,47 \\
7,13\end{array}$ & $\begin{array}{l}9,76 \\
8,87 \\
8,14 \\
7,68 \\
7,33\end{array}$ & $\begin{array}{r}10,02 \\
9,10 \\
8,34 \\
7,86 \\
7,50\end{array}$ & $\begin{array}{r}10,25 \\
9,30 \\
8,52 \\
8,03 \\
7,65\end{array}$ & $\begin{array}{r}10,46 \\
9,49 \\
8,68 \\
8,18 \\
7,79\end{array}$ & $\begin{array}{r}3065 \\
9,65 \\
8.82 \\
8,31 \\
7.91\end{array}$ & $\begin{array}{r}10,83 \\
9,81 \\
8,96 \\
8,44 \\
8,02\end{array}$ & $\begin{array}{r}10,99 \\
9,95 \\
9,08 \\
8,55 \\
8,13\end{array}$ & $\begin{array}{r}11,14 \\
10,08 \\
9,20 \\
8,66 \\
8,23\end{array}$ & $\begin{array}{r}11,29 \\
10,21 \\
9,31 \\
8,76 \\
8,33\end{array}$ & $\begin{array}{r}11,42 \\
10,32 \\
9,42 \\
8,86 \\
8,41\end{array}$ & $\begin{array}{r}11,54 \\
10,43 \\
9,52 \\
8,95 \\
8,50\end{array}$ & $\begin{array}{r}11,66 \\
10,54 \\
9,61 \\
9,03 \\
8,58\end{array}$ \\
\hline $\begin{array}{l}10 \\
11 \\
12 \\
13 \\
14\end{array}$ & $\begin{array}{l}4,48 \\
4,39 \\
4,32 \\
4,26 \\
4,21\end{array}$ & $\begin{array}{l}5,26 \\
5,14 \\
5,04 \\
4,96 \\
4,89\end{array}$ & $\begin{array}{l}\mathbf{5 , 7 7} \\
5,62 \\
5,50 \\
5,40 \\
\mathbf{5 , 3 2}\end{array}$ & $\begin{array}{l}6,14 \\
5,98 \\
5,84 \\
5,73 \\
5,64\end{array}$ & $\begin{array}{l}6,43 \\
6,25 \\
6,10 \\
5,98 \\
5,88\end{array}$ & $\begin{array}{l}6,67 \\
6,47 \\
6,32 \\
6,19 \\
6,08\end{array}$ & $\begin{array}{l}6,88 \\
6,67 \\
6,51 \\
6,37 \\
6,26\end{array}$ & $\begin{array}{l}7,06 \\
6,84 \\
6,67 \\
6,53 \\
6,41\end{array}$ & $\begin{array}{l}7,22 \\
6,99 \\
6,81 \\
6,67 \\
6,54\end{array}$ & $\begin{array}{l}7,36 \\
7,13 \\
6,94 \\
6,79 \\
6,66\end{array}$ & $\begin{array}{l}7,49 \\
7,25 \\
7,06 \\
690 \\
6.77\end{array}$ & $\begin{array}{l}7,60 \\
7,36 \\
7,17 \\
7,01 \\
6,87\end{array}$ & $\begin{array}{l}7,71 \\
7,46 \\
7,26 \\
7,10 \\
6,96\end{array}$ & $\begin{array}{l}7,81 \\
7,56 \\
7,36 \\
7,19 \\
7,05\end{array}$ & $\begin{array}{l}7,91 \\
7,65 \\
7,44 \\
7,27 \\
7,13\end{array}$ & $\begin{array}{l}7,99 \\
7,73 \\
7,52 \\
7,35 \\
7,20\end{array}$ & $\begin{array}{l}8,08 \\
7,81 \\
7,60 \\
7,42 \\
7,27\end{array}$ & $\begin{array}{l}8,15 \\
7,88 \\
7,67 \\
7,49 \\
7,34\end{array}$ & $\begin{array}{l}8,23 \\
7,95 \\
7,73 \\
7,55 \\
7,40\end{array}$ \\
\hline $\begin{array}{l}15 \\
16 \\
17 \\
18 \\
19\end{array}$ & $\begin{array}{l}4,17 \\
4,13 \\
4,10 \\
4,07 \\
4,05\end{array}$ & $\begin{array}{l}4,83 \\
4,78 \\
4,73 \\
4,70 \\
4,66\end{array}$ & $\begin{array}{l}5,25 \\
5,19 \\
5,14 \\
5,09 \\
5,05\end{array}$ & $\begin{array}{l}5,56 \\
5,49 \\
5,43 \\
5,38 \\
5,34\end{array}$ & $\begin{array}{l}5,80 \\
5,72 \\
5,66 \\
5,60 \\
5,55\end{array}$ & $\begin{array}{l}5,99 \\
5,91 \\
5,85 \\
5,79 \\
5,73\end{array}$ & $\begin{array}{l}6,16 \\
6,08 \\
6,01 \\
5,95 \\
5,89\end{array}$ & $\begin{array}{l}6,31 \\
6,22 \\
6,15 \\
6,08 \\
6,02\end{array}$ & $\begin{array}{l}6,44 \\
6,35 \\
6,27 \\
6,20 \\
6,14\end{array}$ & $\begin{array}{l}6,55 \\
6,46 \\
6,38 \\
6,31 \\
6,25\end{array}$ & $\begin{array}{l}6,66 \\
6,56 \\
6,48 \\
6,41 \\
6,34\end{array}$ & $\begin{array}{l}6,76 \\
6,66 \\
6,57 \\
6,50 \\
6,43\end{array}$ & $\begin{array}{l}6,85 \\
6,74 \\
6,66 \\
6,58 \\
6,51\end{array}$ & $\begin{array}{l}6,93 \\
6,82 \\
6,73 \\
6,65 \\
6,58\end{array}$ & $\begin{array}{l}7,00 \\
6,90 \\
6,81 \\
6,73 \\
6,65\end{array}$ & $\begin{array}{l}7,07 \\
6,97 \\
6,87 \\
6,79 \\
6,72\end{array}$ & $\begin{array}{l}7,14 \\
7,03 \\
6,94 \\
6,85 \\
6,78\end{array}$ & $\begin{array}{l}7,20 \\
7,09 \\
7,00 \\
6,9 ! \\
6,81\end{array}$ & $\begin{array}{l}7,26 \\
7,15 \\
7,05 \\
6,97 \\
6,89\end{array}$ \\
\hline $\begin{array}{l}20 \\
24 \\
30 \\
40\end{array}$ & $\begin{array}{l}4,02 \\
3,96 \\
3,89 \\
3,82\end{array}$ & $\begin{array}{l}4,63 \\
4,54 \\
4,45 \\
4,36\end{array}$ & $\begin{array}{l}5,02 \\
4,91 \\
4,80 \\
4,70\end{array}$ & $\begin{array}{l}5,30 \\
5,17 \\
5,05 \\
4,93\end{array}$ & $\begin{array}{l}5,51 \\
5,37 \\
5,24 \\
5,11\end{array}$ & $\begin{array}{l}5,69 \\
5,54 \\
5,40 \\
5,26\end{array}$ & $\begin{array}{l}5,84 \\
5,69 \\
5,53 \\
5,39\end{array}$ & $\begin{array}{l}5,97 \\
5,81 \\
5,65 \\
5,50\end{array}$ & $\begin{array}{l}6,09 \\
5,92 \\
5,76 \\
5,60\end{array}$ & $\begin{array}{l}6,19 \\
6,02 \\
5,85 \\
5,69\end{array}$ & $\begin{array}{l}6,28 \\
6,11 \\
5,93 \\
5,77\end{array}$ & $\begin{array}{l}6,37 \\
6,19 \\
6,01 \\
5,84\end{array}$ & $\begin{array}{l}6,45 \\
6,26 \\
6,08 \\
5,90\end{array}$ & $\begin{array}{l}6,52 \\
6,33 \\
6,14 \\
5,96\end{array}$ & $\begin{array}{l}6,59 \\
6,39 \\
6,20 \\
6,02\end{array}$ & $\begin{array}{l}6,66 \\
6,45 \\
6,26 \\
6,07\end{array}$ & $\begin{array}{l}6.71 \\
6,51 \\
6.31 \\
6,12\end{array}$ & $\begin{array}{l}6,77 \\
6,57 \\
6,36 \\
6,17\end{array}$ & $\begin{array}{l}6,82 \\
6,61 \\
6,41 \\
6,21\end{array}$ \\
\hline $\begin{array}{r}60 \\
120 \\
\infty\end{array}$ & $\begin{array}{l}3,76 \\
3,70 \\
3,64\end{array}$ & $\begin{array}{l}4,28 \\
4,20 \\
4,12\end{array}$ & $\begin{array}{l}4,60 \\
4,50 \\
4,40\end{array}$ & $\begin{array}{l}4,82 \\
4,71 \\
4,60\end{array}$ & $\begin{array}{l}4,99 \\
4,87 \\
4,76\end{array}$ & $\begin{array}{l}5,13 \\
5,00 \\
4,88\end{array}$ & $\begin{array}{l}5,25 \\
5,12 \\
4,99\end{array}$ & $\begin{array}{l}5,36 \\
5,21 \\
5,08\end{array}$ & $\begin{array}{l}5,45 \\
5,30 \\
5,16\end{array}$ & $\begin{array}{l}5,53 \\
5,38 \\
5,23\end{array}$ & $\begin{array}{l}5,60 \\
5,44 \\
5,29\end{array}$ & $\begin{array}{l}5,67 \\
5,50 \\
5,35\end{array}$ & $\begin{array}{l}5,73 \\
5,56 \\
5,40\end{array}$ & $\begin{array}{l}579 \\
5,61 \\
E, 45\end{array}$ & $\begin{array}{l}5,83 \\
5,66 \\
5,49\end{array}$ & $\begin{array}{l}5,88 \\
5,71 \\
5,53\end{array}$ & $\begin{array}{l}5,93 \\
5,75 \\
5,57\end{array}$ & $\begin{array}{l}5,98 \\
5,79 \\
5,61\end{array}$ & $\begin{array}{l}6,01 \\
5,83 \\
5,64\end{array}$ \\
\hline
\end{tabular}

